\title{
Scalar Form Factor of the Pion in the Kroll-Lee-Zumino Field Theory
}

\author{
C. A. Dominguez, ${ }^{1}$ M. Loewe, ${ }^{1,2}$ and M. Lushozi ${ }^{1}$ \\ ${ }^{1}$ Centre for Theoretical \& Mathematical Physics and Department of Physics, University of Cape Town, Rondebosch 7700, South Africa \\ ${ }^{2}$ Instituto de Física, Pontificia Universidad Católica de Chile, Casilla 306, 22 Santiago, Chile
}

Correspondence should be addressed to C. A. Dominguez; cesareo.dominguez@uct.ac.za

Received 28 August 2014; Accepted 4 January 2015

Academic Editor: Alexey A. Petrov

Copyright @ 2015 C. A. Dominguez et al. This is an open access article distributed under the Creative Commons Attribution License, which permits unrestricted use, distribution, and reproduction in any medium, provided the original work is properly cited. The publication of this article was funded by $\mathrm{SCOAP}^{3}$.

\begin{abstract}
The renormalizable Kroll-Lee-Zumino field theory of pions and a neutral rho-meson is used to determine the scalar form factor of the pion in the space-like region at next-to-leading order. Perturbative calculations in this framework are parameter-free, as the masses and the rho-pion-pion coupling are known from experiment. Results compare favorably with lattice QCD calculations.
\end{abstract}

\section{Introduction}

The scalar form factor of the pion [1, 2], and particularly its quadratic radius, plays an important role in chiral perturbation theory (CHPT) [3, 4]. This form factor is defined as the pion matrix element of the QCD scalar current $J_{S}=$ $m_{u} \bar{u} u+m_{d} \bar{d} d$; that is,

$$
F_{S}\left(q^{2}\right)=\left\langle\pi\left(p_{2}\right)\left|J_{S}\right| \pi\left(p_{1}\right)\right\rangle,
$$

where $q^{2}=\left(p_{2}-p_{1}\right)^{2}$. The associated quadratic scalar radius is given by

$$
F_{S}\left(q^{2}\right)=F_{S}(0)\left[1+\frac{1}{6}\left\langle r_{\pi}^{2}\right\rangle_{S} q^{2}+\cdots\right],
$$

where $F_{S}(0)$ is the pion sigma term

$$
F_{S}(0) \equiv \sigma_{\pi}=m_{q} \frac{\partial M_{\pi}^{2}}{\partial m_{q}} .
$$

The scalar radius fixes $\bar{l}_{4}$, one of the low energy constants of CHPT, through the relation

$$
\left\langle r_{\pi}^{2}\right\rangle_{S}=\frac{3}{8 \pi^{2} F_{\pi}^{2}}\left[\bar{\ell}_{4}-\frac{13}{12}+O\left(M_{\pi}^{2}\right)\right],
$$

where $F_{\pi}=91.9 \pm 0.1 \mathrm{MeV}$ is the physical pion decay constant [5]. The low energy constant $\bar{\ell}_{4}$, in turn, determines the leading contribution in the chiral expansion of the pion decay constant; that is,

$$
\frac{F_{\pi}}{F}=1+\left(\frac{M_{\pi}}{4 \pi F_{\pi}}\right)^{2} \bar{\ell}_{4}+O\left(M_{\pi}^{4}\right),
$$

where $F$ is the pion decay constant in the chiral limit.

This scalar form factor is not accessible experimentally, but it has been determined from lattice QCD (LQCD) [6-8], or hadronic models $[9,10]$.

Theoretically, the ideal tool to study this form factor, independently from LQCD, is the Kroll-Lee-Zumino Abelian renormalizable field theory of pions and a neutral $\rho$-meson $[11,12]$. This provides the appropriate field theory platform for the phenomenological vector meson dominance (VMD) model $[13,14]$, allowing for a systematic calculation of higher order quantum corrections ([15], this paper, has a misprint in equation (15) (the sign of the first term in curly brackets should be negative), with the remaining equations being correct. The electromagnetic square radius of the pion quoted in the paper is incorrect; the correct value is $\left\langle r_{\pi}^{2}\right\rangle_{\mathrm{EM}}=$ $0.46 \mathrm{fm}^{2}$, in much better agreement with data than naive (single $\rho$ ) VMD.) [16]. Due to the renormalizability of the theory, predictions are parameter-free, as the strong $\rho \pi \pi$ 
coupling, $g_{\rho \pi \pi}$, is known from experiment. In spite of this coupling being a strong interaction quantity, perturbative calculations in the $\overline{M S}$ scheme make sense because the effective expansion parameter turns out to be $\left(g_{\rho \pi \pi} / 4 \pi\right)^{2} \simeq$ 0.2 .

The KLZ theory has been used to compute the nextto-leading order (NLO) correction to the tree level (VMD) electromagnetic form factor of the pion in the space-like region with very good results [15]. In fact, it agrees with data up to $q^{2} \simeq-10 \mathrm{GeV}^{2}$ with a chi-squared per degree of freedom $\chi_{F}^{2}=1.1$, as opposed to VMD which gives $\chi_{F}^{2}=5.0$. In addition, the mean-squared radius at NLO is $\left\langle r_{\pi}^{2}\right\rangle=0.46 \mathrm{fm}^{2}$, compared with the experimental result [5] $\left\langle r_{\pi}^{2}\right\rangle=0.45 \pm 0.01 \mathrm{fm}^{2}$, and the VMD value $\left\langle r_{\pi}^{2}\right\rangle=0.39 \mathrm{fm}^{2}$.

In this note we compute in this framework the scalar form factor of the pion at NLO in the space-like region and compare with current results from LQCD.

The KLZ Lagrangian is given by

$$
\begin{aligned}
\mathscr{L}_{\mathrm{KLZ}}= & \partial_{\mu} \phi \partial^{\mu} \phi^{*}-M_{\pi}^{2} \phi \phi^{*}-\frac{1}{4} \rho_{\mu \nu} \rho^{\mu \nu}+\frac{1}{2} M_{\rho}^{2} \rho_{\mu} \rho^{\mu} \\
& +g_{\rho \pi \pi} \rho_{\mu} J_{\pi}^{\mu}+g_{\rho \pi \pi}^{2} \rho_{\mu} \rho^{\mu} \phi \phi^{*},
\end{aligned}
$$

where $\rho_{\mu}$ is a vector field describing the $\rho^{0}$ meson $\left(\partial_{\mu} \rho^{\mu}=0\right)$, $\phi$ is a complex pseudoscalar field describing the $\pi^{ \pm}$mesons, $\rho_{\mu \nu}$ is the usual field strength tensor: $\rho_{\mu \nu}=\partial_{\mu} \rho_{\nu}-\partial_{\nu} \rho_{\mu}$, and $J_{\pi}^{\mu}$ is the $\pi^{ \pm}$current: $J_{\pi}^{\mu}=i \phi^{*} \overleftrightarrow{\partial_{\mu}} \phi$. In spite of the explicit presence of the $\rho^{0}$ mass term in the Lagrangian, the theory is renormalizable because the neutral vector meson is coupled to a conserved current [11, 12]. Figures 1 and 2 show, respectively, the LO and the NLO diagrams, where the cross indicates the coupling of the current to the two pions. Notice that while the Lagrangian (6) contains a $\rho \rho \pi \pi$ quartic coupling, this term only contributes in this application at NNLO and beyond.

Using the Feynman propagator for the $\rho$-meson, and in $d$ dimensions, the unrenormalized vertex function in Figure 2 in dimensional regularization is given by

$$
\begin{aligned}
\widetilde{G}\left(q^{2}\right)= & -2 \frac{g_{\rho \pi \pi}^{2}}{(4 \pi)^{2}}\left(\mu^{2}\right)^{(2-(d / 2))} \int_{0}^{1} d x_{1} \int_{0}^{1-x_{1}} d x_{2} \\
& \times\left\{\frac{2}{\varepsilon}-\ln \left(\frac{\Delta\left(q^{2}\right)}{\mu^{2}}\right)-\frac{1}{2}-\gamma+\ln (4 \pi)+\frac{1}{2 \Delta\left(q^{2}\right)}\right. \\
& \cdot\left[M_{\pi}^{2}\left(x_{1}+x_{2}-2\right)^{2}-q^{2}\left(x_{1} x_{2}-x_{1}-x_{2}+2\right)\right] \\
& +O(\varepsilon)\},
\end{aligned}
$$

where $\Delta\left(q^{2}\right)$ is defined as

$$
\Delta\left(q^{2}\right)=M_{\pi}^{2}\left(x_{1}+x_{2}\right)^{2}+M_{\rho}^{2}\left(1-x_{1}-x_{2}\right)-x_{1} x_{2} q^{2} .
$$

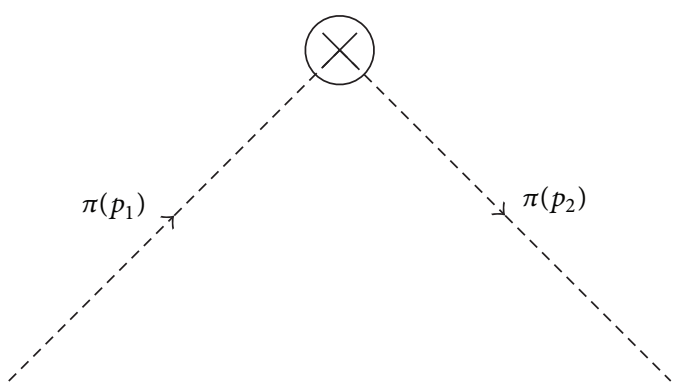

FIGURE 1: Leading order (LO) contribution to the scalar form factor of the pion. The cross indicates the coupling of the scalar current to two pions.

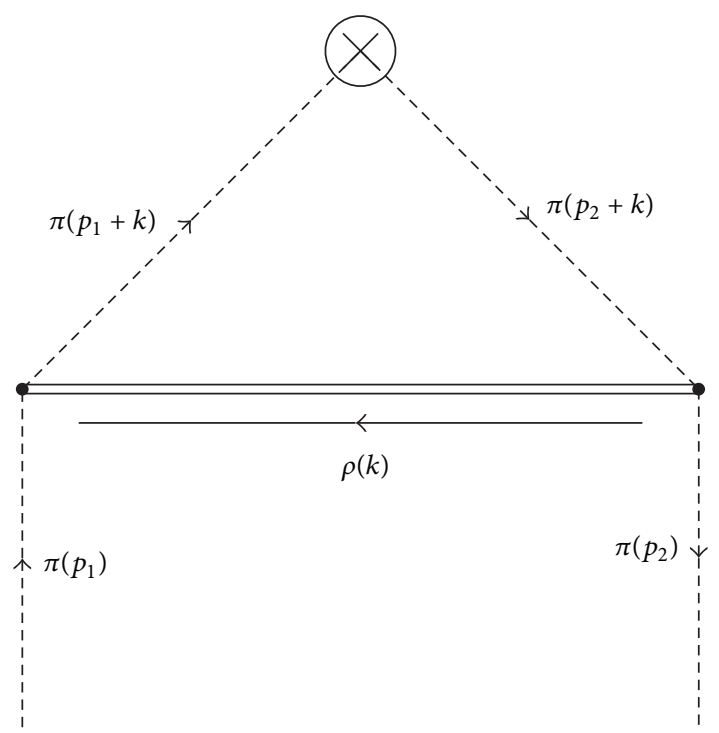

FIGURE 2: Next-to-leading order (NLO) contribution to the scalar form factor.

In the $\overline{M S}$ scheme and renormalizing the vertex function at the point $q^{2}=0$, the NLO contribution in Figure 2 is [16]

$$
\begin{aligned}
& G\left(q^{2}\right)-G(0) \\
& =-2 \frac{g_{\rho \pi \pi}^{2}}{(4 \pi)^{2}} \int_{0}^{1} d x_{1} \int_{0}^{1-x_{1}} d x_{2} \\
& \quad \times\left\{\ln \left(\frac{\Delta\left(q^{2}\right)}{\Delta(0)}\right)+\frac{1}{2}\left[M_{\pi}^{2}\left(x_{1}+x_{2}-2\right)^{2}\right.\right. \\
& \cdot\left(\frac{1}{\Delta\left(q^{2}\right)}-\frac{1}{\Delta(0)}\right)-\frac{q^{2}}{\Delta\left(q^{2}\right)} \\
& \left.\left.\cdot x_{1} x_{2}-x_{1}-x_{2}+2\right]\right\},
\end{aligned}
$$

with

$$
F_{S}\left(q^{2}\right)=F_{S}(0)\left[1+G\left(q^{2}\right)-G(0)\right]
$$




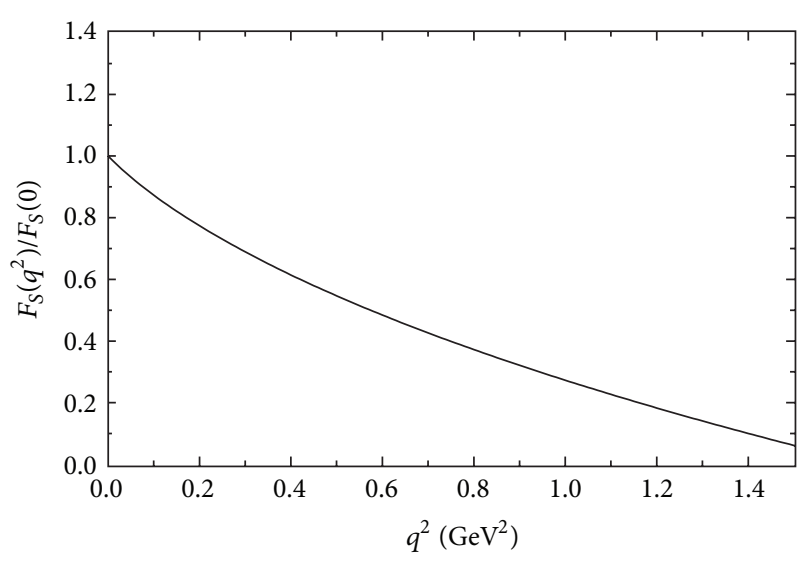

FIgURE 3: The normalized scalar form factor (8), to NLO in the space-like region.

For details on the renormalization procedure for the fields, masses, and coupling, see [15]. The result of a numerical evaluation of (9), using $g_{\rho \pi \pi}^{2}=36.0 \pm 0.2$ from the measured width of the $\rho$-meson [5], is shown in Figure 3. Regarding the scalar radius, defined in (2), we confirm the NLO result obtained in [16]

$$
\left\langle r_{\pi}^{2}\right\rangle_{S}=0.4 \mathrm{fm}^{2}
$$

with a negligible error due to the strong coupling.

This value is smaller than typical values in the literature [6-10]. However, it must be kept in mind that the NLO result is expected to be a lower bound; that is, with $\left[G\left(q^{2}\right)-\right.$ $G(0)]<0$, the NNLO would reduce $F_{S}\left(q^{2}\right)$, thus increasing the radius. A rough order of magnitude estimate of the size of the NNLO contribution suggests a correction of some $20 \%$ to the NLO term (the NNLO calculation is quite formidable and beyond the scope of this note). This is obtained by estimating a typical two-loop diagram, for example, the $\rho$ meson propagator at NNLO and comparing it with the NLO result. The Feynman integrals in the variables $x_{i}$ at NLO and NNLO are of order $\mathcal{O}(1)$ in the $q^{2}$ range explored here. We find the total contribution from this diagram to be over $20 \%$ of the NLO, thus increasing the radius to $\left\langle r_{\pi}^{2}\right\rangle_{S} \simeq 0.5 \mathrm{fm}^{2}$. It should be clear that this result (11) would be affected by (unknown) systematic uncertainties arising, for example, from hadronic contributions from fields absent from the KLZ Lagrangian (6).

A comparison of the KLZ form factor itself at low $\left|q^{2}\right|<$ $0.5 \mathrm{GeV}^{2}$ with LQCD results read from figures in $[6,8]$ shows good agreement. It should be mentioned, though, that LQCD results from [6] are for light-quark masses in the range from $m_{s} / 6$ to $m_{s} / 2$, while those from [8] are for $m_{\pi}=325 \mathrm{MeV}$. These LQCD determinations find values for the scalar radius higher than in this analysis (11); that is, $\left\langle r_{\pi}^{2}\right\rangle_{S}=0.6 \pm 0.1 \mathrm{fm}^{2}$ from [6] and $\left\langle r_{\pi}^{2}\right\rangle_{S}=0.637 \pm 0.023 \mathrm{fm}^{2}$ from [8]. These results for the radius are determined from, for example, chiral extrapolations to the physical pion mass. Our results for the form factor are also in agreement within less than $10 \%$ with a CHPT calculation [17] in the range $-q^{2}=0-0.2 \mathrm{GeV}^{2}$.
Regarding potential contributions to the form factor and its radius, for example, from other scalar degrees of freedom absent from the KLZ Lagrangian, they should be understood as part of the systematic uncertainties. The excellent agreement with data of KLZ results for the electromagnetic pion form factor [15] might be a result of the absence of other hadronic contributions with the same quantum numbers as the neutral $\rho$-meson. In the case of the scalar form factor, these additional contributions are potentially present, and thus could become part of the systematic uncertainties.

\section{Conflict of Interests}

The authors declare that there is no conflict of interests regarding the publication of this paper.

\section{Acknowledgments}

This work was supported in part by FONDECyT (Chile) under Grants 1130056 and 1120770, by NRF (South Africa), and by the University of Cape Town URC. The authors wish to thank Gary Tupper for valuable discussions on KLZ and Hartmut Wittig for enlightening exchanges on LQCD.

\section{References}

[1] T. N. Truong and R. S. Willey, "Branching ratios for decays of light Higgs bosons," Physical Review D, vol. 40, no. 11, pp. 36353640, 1989.

[2] J. F. Donoghue, J. Gasser, and H. Leutwyler, "The decay of a light Higgs boson," Nuclear Physics B, vol. 343, no. 2, pp. 341-368, 1990.

[3] S. Scherer, "Introduction to chiral perturbation theory," Advances in Nuclear Physics, vol. 27, p. 277, 2003.

[4] J. Gasser, "Light-quark dynamics," in Lectures on Flavor Physics, vol. 629 of Lecture Notes in Physics, pp. 1-35, Springer, Berlin, Germany, 2004.

[5] J. Beringer, J. F. Arguin, R. M. Barnett et al., "Review of particle physics," Physical Review D, vol. 86, Article ID 010001, 2012.

[6] S. Aoki, T. W. Chiu, H. Fukaya et al., "Pion form factors from two-flavor lattice QCD with exact chiral symmetry," Physical Review D, vol. 80, Article ID 034508, 2009.

[7] S. Aoki, Y. Aoki, C. Bernard et al., "Review of lattice results concerning low energy particle physics," http://arxiv.org/abs/1310 .8555 .

[8] V. Gülpers, G. von Hippel, and H. Wittig, "The scalar pion form factor in two-flavor lattice QCD," Physical Review D, vol. 89, Article ID 094503, 2014.

[9] J. A. Oller and L. Roca, "Scalar radius of the pion and zeros in the form factor," Physics Letters B, vol. 651, no. 2-3, pp. 139-146, 2007.

[10] L. Roca, J. A. Oller, and C. Schat, "Scalar radius of the pion and $\gamma \gamma \rightarrow \pi \pi$," PoS EFT, vol. 09, p. 031, 2009.

[11] N. M. Kroll, T. D. Lee, and B. Zumino, "Neutral vector mesons and the hadronic electromagnetic current," Physical Review, vol. 157, no. 5, pp. 1376-1399, 1967.

[12] J. H. Lowenstein and B. Schroer, "Gauge invariance and ward identities in a massive-vector-meson model," Physical Review D, vol. 6, no. 6, pp. 1553-1571, 1972. 
[13] J. J. Sakurai, "Theory of strong interactions," Annals of Physics, vol. 11, no. 1, pp. 1-48, 1960.

[14] J. J. Sakurai, Currents and Mesons, University of Chicago Press, Chicago, Ill, USA, 1969.

[15] C. A. Dominguez, J. I. Jottar, M. Loewe, and B. Willers, "Pion form factor in the Kroll-Lee-Zumino model," Physical Review D, vol. 76, no. 9, Article ID 095002, 2007.

[16] C. A. Dominguez, M. Loewe, and B. Willers, "Scalar radius of the pion in the Kroll-Lee-Zumino renormalizable theory," Physical Review D, vol. 78, Article ID 057901, 2008.

[17] A. Jüttner, "Revisiting the pion's scalar form factor in chiral perturbation theory," Journal of High Energy Physics, vol. 2012, no. 1, article 7, 2012. 

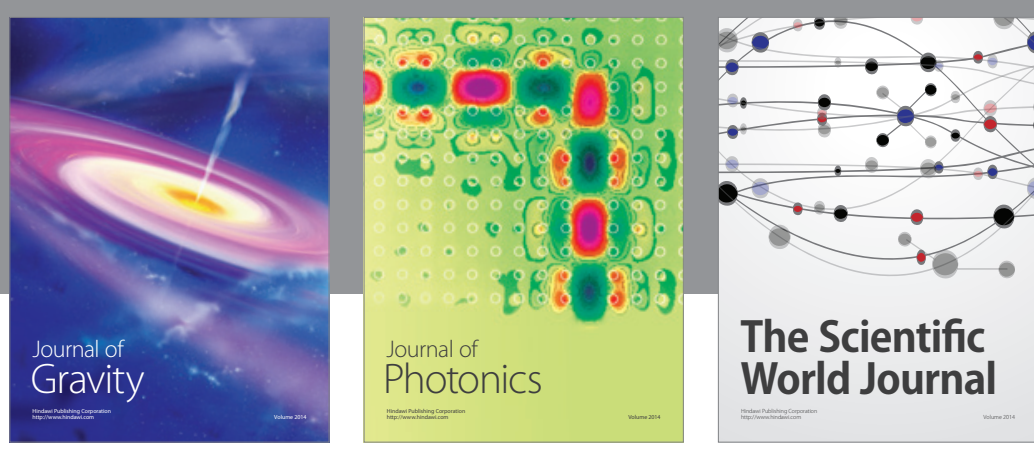

The Scientific World Journal
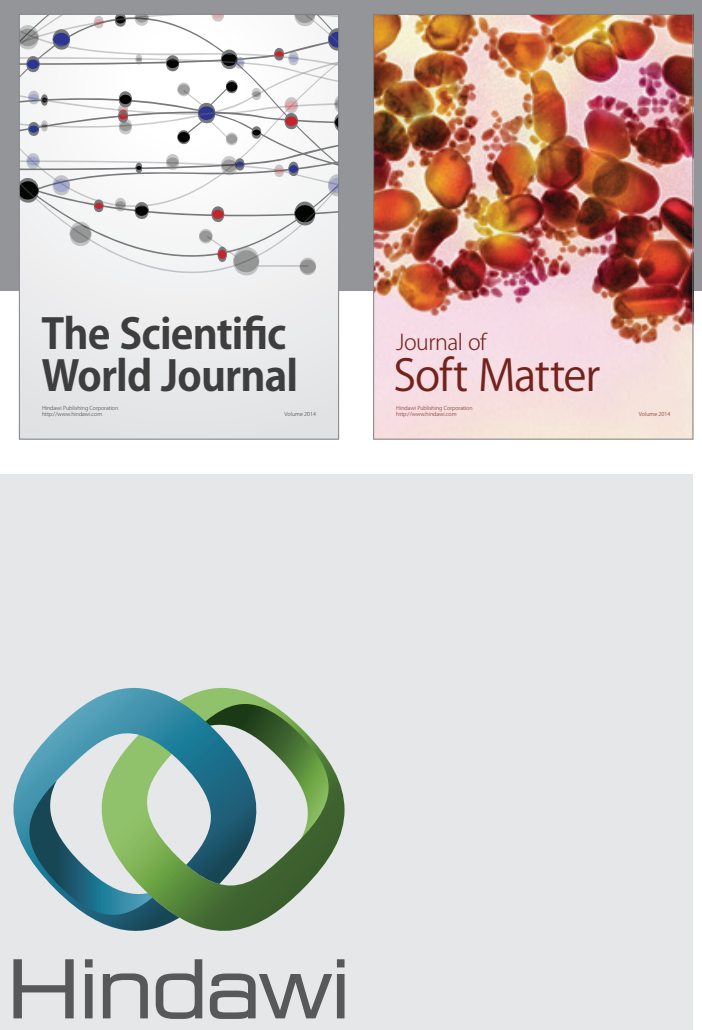

Submit your manuscripts at

http://www.hindawi.com

nternational Journal of

Statistical Mechanics
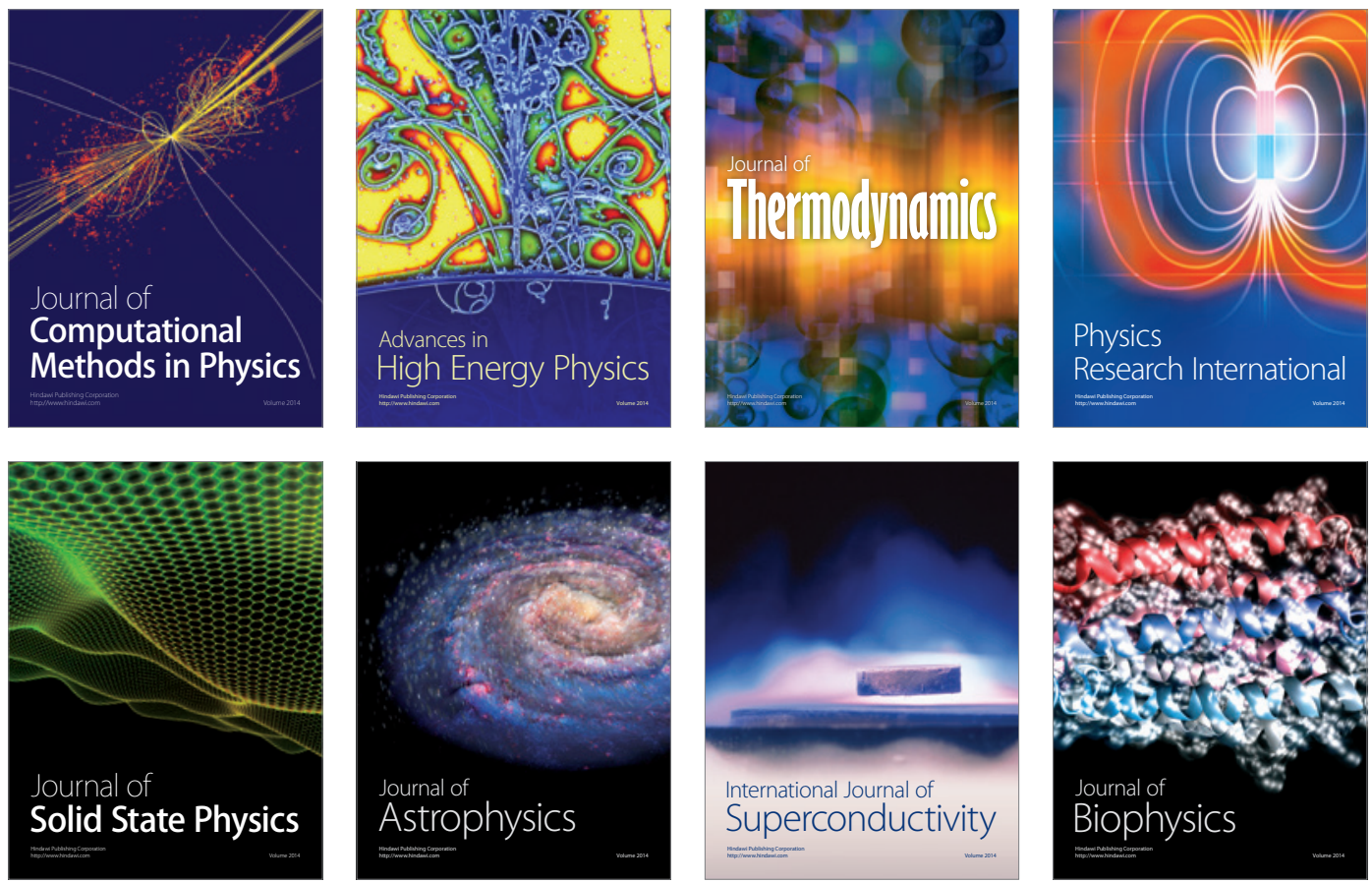
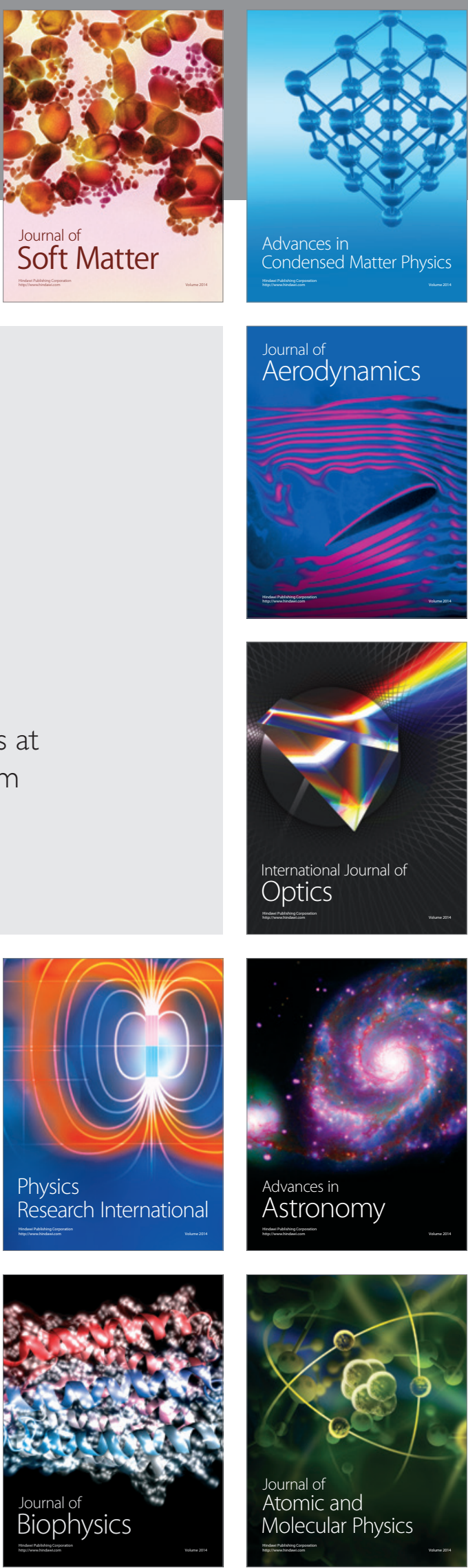